\title{
A solution to Comfort's question on the countable compactness of powers of a topological group
}

\author{
by \\ Artur Hideyuki Tomita (São Paulo) \\ Dedicated to Professor Edson Farah \\ on the ocassion of his 90th birthday
}

\begin{abstract}
In 1990, Comfort asked Question 477 in the survey book "Open Problems in Topology": Is there, for every (not necessarily infinite) cardinal number $\alpha \leq 2^{\mathfrak{c}}$, a topological group $G$ such that $G^{\gamma}$ is countably compact for all cardinals $\gamma<\alpha$, but $G^{\alpha}$ is not countably compact?

Hart and van Mill showed in 1991 that $\alpha=2$ answers this question affirmatively

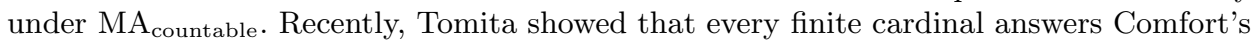
question in the affirmative, also from MA countable. However, the question has remained open for infinite cardinals.

We show that the existence of $2^{\mathfrak{c}}$ selective ultrafilters $+2^{\mathfrak{c}}=2^{<2^{\mathfrak{c}}}$ implies a positive answer to Comfort's question for every cardinal $\kappa \leq 2^{\mathfrak{c}}$. Thus, it is consistent that $\kappa$ can be a singular cardinal of countable cofinality. In addition, the groups obtained have no non-trivial convergent sequences.
\end{abstract}

1. Introduction. Throughout this paper, $\omega^{*}$ will denote the set of all free ultrafilters on $\omega$. Given a set $A$ and a cardinal $\kappa,[A]^{<\kappa}$ will denote the set of all subsets of $A$ of size $<\kappa$ and $[A]^{\kappa}$ will denote the set of all subsets of $A$ of size $\kappa$.

Martin's Axiom restricted to countable posets $\left(\mathrm{MA}_{\text {countable }}\right)$ is a weaker form of Martin's Axiom (MA). MA countable is equivalent to the statement "the intersection of fewer than $\mathfrak{c}$ dense open sets of the real line is dense" (see [15]). MA implies $\mathrm{MA}_{\text {countable }}$ and $\mathrm{MA}_{\text {countable }}$ implies the existence of selective ultrafilters. The reverse implications are not true.

2000 Mathematics Subject Classification: Primary 54B10, 54A35; Secondary 22A05, 03E50, 54G20.

Key words and phrases: $p$-limit, countably compact group, topological product, selective ultrafilters, Open Problems in Topology, convergent sequences, Martin's Axiom, Comfort's question. 
The reader not familiar with selective ultrafilters can replace "the existence of $2^{\mathfrak{c}}$ selective ultrafilters" by MA or CH.

The study of the non-productivity of countable compactness in topological spaces started in the 1950's, when Terasaka [20] and Novák [17] showed that there exists a countably compact space whose square is not pseudocompact. The finite and the countable cases were studied by Frolík in the 1960's and the infinite case by Saks in the 1970's.

Scarborough and Stone [19] showed in 1966 that if $\left\{X_{i}: i \in I\right\}$ is a family of topological spaces then $\prod_{i \in I} X_{i}$ is countably compact if and only if $\prod_{i \in J} X_{i}$ is countably compact for each $J \subseteq I$ with $|J| \leq 2^{2^{c}}$.

Ginsburg and Saks [13] improved the theorem above in 1975. They showed that $\prod_{i \in I} X_{i}$ is countably compact if and only if $\prod_{i \in J} X_{i}$ is countably compact for each $J \subseteq I$ with $|J| \leq 2^{\text {c }}$. A natural question is whether $2^{\mathfrak{c}}$ could be replaced by a smaller cardinal.

Saks [18] showed in 1979 that $2^{\mathfrak{c}}$ was consistently the best possible. He showed that if there exist $2^{\mathfrak{c}}$ selective ultrafilters then there exists a countably compact space $X$ such that $X^{\alpha}$ is countably compact for every $\alpha<2^{\mathfrak{c}}$ but $X^{2^{\mathrm{c}}}$ is not.

Comfort and Ross [6] showed in 1966 that the product of pseudocompact groups is pseudocompact. That motivated Comfort to ask whether countable compactness is productive in the class of topological groups.

E. van Douwen [9] answered this question in the negative in 1980. Indeed, he produced two countably compact groups whose product is not countably compact from MA. His construction is divided into two parts:

(1) (MA) There exists an infinite countably compact group without nontrivial convergent sequences inside the compact group $2^{\mathfrak{c}}$.

(2) (ZFC) A countably compact group of order 2 without non-trivial convergent sequences contains two countably compact subgroups whose product is not countably compact.

At the end of [9], van Douwen wrote that he was able to produce a single countably compact group whose square is not countably compact. He wrote he would publish this result elsewhere but he never did.

Hart and van Mill [15] showed in 1991 that there exists a countably compact topological group whose square is not countably compact under $\mathrm{MA}_{\text {countable }}$. The example contains non-trivial convergent sequences as it makes use of $\omega$-bounded groups in the construction.

Koszmider, Tomita and Watson [16] showed in 2000 that $\mathrm{MA}_{\text {countable }}$ implies the existence of a countably compact group without non-trivial convergent sequences whose square is not countably compact.

Dikranjan and Tkachenko [8] showed in 2003 that, under MA, every group of size $\mathfrak{c}$ that admits a countably compact group topology also ad- 
mits a countably compact group topology without non-trivial convergent sequences.

Recently, Garcia-Ferreira, Tomita and Watson [12] showed that the existence of a selective ultrafilter $p$ implies the existence of an infinite $p$-compact group of order 2 without non-trivial convergent sequences. In particular, the existence of a selective ultrafilter implies the existence of an infinite countably compact group of order 2 without non-trivial convergent sequences. Applying results from [9] and [1], the authors of [12] showed that the nonproductivity of countable compactness does not depend on some form of Martin's Axiom.

W. W. Comfort asked in 1990 the following question in Open Problems in Topology:

Question 1.1 ([4, Question 477]). Is there, for every (not necessarily infinite) cardinal number $\alpha \leq 2^{\mathfrak{c}}$, a topological group $G$ such that $G^{\gamma}$ is countably compact for all cardinals $\gamma<\alpha$, but $G^{\alpha}$ is not countably compact?

According to Comfort, the motivation for his question came from two results:

(1) Hart and van Mill [15]: There exists a countably compact group whose square is not countably compact from $\mathrm{MA}_{\text {countable }}$

(2) Ginsburg and Saks [13]: If the $2^{\mathfrak{c}}$ th power of a topological space $X$ is countably compact then every power of $X$ is countably compact.

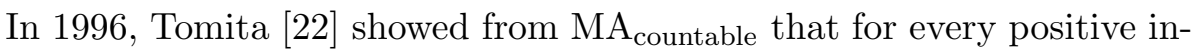
teger $k$ there exists a topological group $G$ such that $G^{k}$ is countably compact but $G^{2^{k}}$ is not. In 1999, Tomita [23] showed from MA countable that there is a topological group whose square is countably compact but whose cube is not. Recently, Tomita [25] showed that, under $\mathrm{MA}_{\text {countable, there exists for each }}$ positive integer $k$ a topological group $H$ such that $H^{k}$ is countably compact but $H^{k+1}$ is not. Thus, each finite cardinal answers Comfort's question affirmatively under $\mathrm{MA}_{\text {countable }}$.

Yang [28] showed in 1985 that the following are equivalent:

(1) there exists a space $X$ such that $X^{\alpha}$ is countably compact for each $\alpha<2^{\mathfrak{c}}$ but $X^{2^{\mathfrak{c}}}$ is not countably compact;

(2) for every family $\mathcal{U} \subseteq \omega^{*}$ of size less than $2^{\mathfrak{c}}$ there exists $p \in \omega^{*}$ such that $p$ is not Rudin-Keisler compatible with any element of $\mathcal{U}$.

In 2000, in a personal communication, M. Hrušak showed that the second statement in the equivalence above is not true in a model due to Shelah [3]. Thus, it is consistent that $2^{\mathfrak{c}}$ does not answer Comfort's question in the affirmative.

Garcia-Ferreira and Tomita [11] showed in 2003 that Ginsburg and Saks' theorem is consistently the best possible for the class of topological groups. 
They showed, via forcing, the existence of a family $\left\{G_{\xi}: \xi<2^{\mathfrak{c}}\right\}$ of topological groups such that $\prod_{\xi \in A} G_{\xi}$ is countably compact if and only if $|A|<2^{\mathfrak{c}}$. The groups in that example cannot be the same. Thus, they are not relevant to Question 477.

In this note, we will show that it is consistent that each cardinal not greater than $2^{\mathfrak{c}}$ answers Comfort's question affirmatively. The examples follow from the existence of $2^{\mathfrak{c}}$ selective ultrafilters and $2^{<2^{\mathfrak{c}}}=2^{\mathfrak{c}}$. In Section 2 , we will review spaces defined by $p$-limits and ultraproducts. In Section 3, we will present the tools to produce countably compact groups using selective ultrafilters. In Section 4, we will show that the existence of $\mathfrak{c}$ selective ultrafilters implies that, for each positive integer $n$, there exists a topological group $G$ such that $G^{n}$ is countably compact but $G^{n+1}$ is not. As a corollary, we improve the forcing example from Garcia-Ferreira and Tomita [11] about quasi $M$-compact groups. In Section 5 , we will present a solution to Comfort's question for infinite cardinals.

2. Ultraproducts and spaces defined by p-limits. In 1970, Bernstein introduced the following concept that is very useful in the construction of countably compact spaces in products:

Definition $2.1([2])$. Given a free ultrafilter $p \in \omega^{*}$ and a topological space $X$, we say that a point $x \in X$ is the $p$-limit of $\left\{x_{n}: n \in \omega\right\} \subseteq X$ if for every neighbourhood $U$ of $x$ the set $\left\{n \in \omega: x_{n} \in U\right\}$ is an element of $p$.

For a Tikhonov space $X, x$ is the $p$-limit of a sequence $\left\{x_{n}: n \in \omega\right\}$ if and only if $\beta f(p)=x$, where $\beta f: \beta \omega \rightarrow \beta X$ is the Cech-Stone extension of the function $f: \omega \rightarrow X$ defined by $f(n)=x_{n}$ for each $n \in \omega$.

If $x$ is an accumulation point of a sequence then there is an ultrafilter $p$ for which $x$ is the $p$-limit of this sequence. This motivated introducing the following class of spaces:

Definition $2.2([2])$. Given a free ultrafilter $p \in \omega^{*}$ and a topological space $X$, we say that $X$ is $p$-compact if every sequence in $X$ has a $p$-limit.

Every $p$-compact space is countably compact. For a fixed ultrafilter $p$, $p$-compactness is a productive property. The proof of productivity of $p$ compactness is similar to the proof of Tikhonov's theorem: a sequence in a product of spaces has a $p$-limit if and only if every projection of the sequence has a $p$-limit. It follows that every power of a $p$-compact space is countably compact.

The examples that solve Comfort's question cannot be $p$-compact for any $p$. However, $p$-compactness will be used in the construction of the examples in Section 5. We will produce a larger group in which all small subsets are contained in small $p$-compact groups for some ultrafilter $p$. Our examples in Section 5 will be subgroups of this larger group. 
Garcia-Ferreira [10] introduced in 1993 another class of spaces that depend on $p$-limits.

Definition 2.3 (Garcia-Ferreira). Let $\emptyset \neq M \subseteq \omega^{*}$. We say that a space $X$ is quasi $M$-compact if every sequence in $X$ has a $p$-limit in $X$ for some $p \in M$.

In the definition above, $M$ can be viewed as a set of ultrafilters that witnesses the countable compactness of a space. For example, $p$-compactness is equivalent to quasi $\{p\}$-compactness and countable compactness is equivalent to quasi $\omega^{*}$-compactness.

Garcia-Ferreira and Tomita [11] showed that it is consistent that some countably compact groups need a large number of witnesses for their countable compactness. Indeed, they showed via forcing that there exists a countably compact group that is not quasi $M$-compact for any $M$ of size $<2^{\mathfrak{c}}$. We will improve this example in Section 4.

The ultrapower $\left([\mathfrak{c}]^{<\omega}\right)^{\omega} / p$ has been applied in [12], [27] and [26] to obtain $p$-compact groups with special properties. In our examples, the groups have size $\kappa=\mathfrak{c}$ or $\kappa=2^{\mathfrak{c}}$.

Definition 2.4. Given a cardinal $\kappa$ and $p \in \omega^{*}$, for each $f \in\left([\kappa]^{<\omega}\right)^{\omega}$, define $[f]_{p}=\left\{g \in\left([\kappa]^{<\omega}\right)^{\omega}:\{n \in \omega: f(n)=g(n)\} \in p\right\}$. Denote by $\left([\kappa]^{<\omega}\right)^{\omega} / p=\left\{[f]_{p}: f \in\left([\kappa]^{<\omega}\right)^{\omega}\right\}$ the vector space over 2 under the operation $[f]_{p} \triangle[g]_{p}:=[f \triangle g]_{p}$, where $(f \triangle g)(n)=f(n) \backslash g(n) \cup g(n) \backslash f(n)$. If $f$ is the constant function $\{\mu\}$, we will denote $[f]_{p}$ by $[\vec{\mu}]_{p}$.

Lemma 2.5. Let $p$ be an element of $\omega^{*}$ and $\kappa$ be an infinite cardinal.

(a) The set $\left([\kappa]^{<\omega}\right)^{\omega} / p$ under the operation $\triangle$ is a vector space over the field 2 .

(b) A family $\left\{\left[g_{\alpha}\right]_{p}: \alpha \in I\right\}$ of $p$-equivalence classes of $\left([\kappa]^{<\omega}\right)^{\omega} / p$ :

(i) is linearly independent if and only if for every $F \in[I]^{<\omega}$ there exists $A \in p$ such that $\Delta\left(\left\{g_{\alpha}(n): \alpha \in F\right\}\right) \neq \emptyset$ for all $n \in A$;

(ii) generates the vector space $\left([\kappa]^{<\omega}\right)^{\omega} / p$ if and only if for every $g \in\left([\kappa]^{<\omega}\right)^{\omega}$ there exist $A \in p$ and $F \in[I]^{<\omega}$ such that $g(n)=$ $\Delta\left(\left\{g_{\alpha}(n): \alpha \in F\right\}\right)$ for all $n \in A$.

The next theorem connects ultrapowers on $p$ to $p$-compactness. The proof for $\kappa=\mathfrak{c}$ appears in [27]. The proof for $\kappa=\kappa^{\omega}$ requires only relabelling $\mathfrak{c}$ as $\kappa$. We will omit it.

Theorem 2.6. Let $\kappa=\kappa^{\omega}$ be an infinite cardinal. Let $\Phi:[\kappa]^{<\omega} \rightarrow K$ be a group homomorphism with $K$ a topological group and $\left\{g_{\alpha}: \alpha \in \kappa\right\}$ be a family of functions from $\omega$ into $[\kappa]^{<\omega}$. If $\left\{\left[g_{\alpha}\right]: \alpha \in \kappa\right\} \cup\{[\vec{\alpha}]: \alpha<\kappa\}$ generates $\left([\kappa]^{<\omega}\right)^{\omega} / p$ and $\left\{\Phi\left(g_{\alpha}(n)\right): n \in \omega\right\}$ has a $p$-limit in $\Phi\left([\kappa]^{<\omega}\right)$ for each $\alpha<\kappa$ then $\Phi([\kappa]<\omega)$ is p-compact. 
3. Selective ultrafilters. The construction of a countably compact group topology on a group $G$ usually involves the topology generated by a family of group homomorphisms from $G$ into a compact group.

The Continuum Hypothesis or some form of Martin's Axiom are used in [8], [14]-[16], [21]-[23] and [25] to produce group homomorphisms. A countably closed forcing in a $\mathrm{CH}$ model was used in [7], [11], [16], [24] and [26] to produce group homomorphisms.

In [12], group homomorphisms involving $p$-limits are constructed from the existence of a single selective ultrafilter $p$. In [27], group homomorphisms involving $p$-limits and $q$-limits are constructed from the existence of two incomparable selective ultrafilters $p$ and $q$. For the construction in this paper, we need to deal with $2^{\mathfrak{c}}$ selective ultrafilters.

Our goal in this section is to prove Lemma 3.7. This lemma gives some combinatorial properties of infinitely many incomparable selective ultrafilters. These properties will be used to show the existence of enough group homomorphisms (Lemmas 4.1 and 5.1).

The reader interested mainly in the construction of countably compact groups can skip the proofs in this section. Only the statement of Lemma 3.7 will be used in the following sections.

The definition of a selective ultrafilter is related to the Rudin-Keisler order.

Definition 3.1. Given $p, q \in \omega^{*}$, we say that $p \leq_{\mathrm{RK}} q$ if there exists a function $f: \omega \rightarrow \omega$ such that $\beta f(q)=p$, where $\beta f$ is the Cech-Stone extension of $f$. The pre-order $\leq_{\mathrm{RK}}$ on $\omega^{*}$ is called the Rudin-Keisler order.

LEMma 3.2. Two ultrafilters $p$ and $q$ are Rudin-Keisler equivalent if and only if there exists a bijection $f: \omega \rightarrow \omega$ such that $\beta f(p)=q$.

More on selective ultrafilters can be found in [5]:

Lemma 3.3. The following are equivalent for $p \in \omega^{*}$ :

(i) $p$ is Rudin-Keisler minimal;

(ii) $p$ is selective, that is, if $\left\{P_{n}: n \in \omega\right\}$ is a partition of $\omega$ then either there is $m \in \omega$ such that $P_{m} \in p$ or there is $B \in p$ such that $\left|B \cap P_{n}\right|=1$ for each $n \in \omega$.

Every selective ultrafilter $p$ is a $P$-point, that is, if $\left\{A_{n}: n \in \omega\right\} \subseteq p$ then there exists $A \in p$ such that $A \backslash A_{n}$ is finite for each $n \in \omega$.

The existence of selective ultrafilters is independent of the usual axioms of set theory. There are $2^{\mathfrak{c}}$ selective ultrafilters under $\mathrm{CH}$ or MA. Shelah showed via forcing that there are models with no $P$-points (in particular, no selective ultrafilters). Kunen showed that there are no selective ultrafilters in the random real model. 
Lemmas 3.4 and 3.5 stated below give some combinatorial properties for a selective ultrafilter and a pair of selective ultrafilters. The first lemma was used in [12] to show the existence of a compact group without non-trivial convergent sequence from a selective ultrafilter. The second lemma was used in [27] to show that the Comfort order is not downward directed from the existence of two selective ultrafilters.

Lemma 3.6 is the countable version of Lemma 3.5. The proofs of Lemmas 3.4 and 3.5 appear in [27] and are omitted. We need only the statements of these lemmas to prove Lemma 3.6.

LEMMA 3.4 ([12], [27]). Let $p$ be a selective ultrafilter and $\left\{a_{k}: k \in \omega\right\} \in p$ be a strictly increasing sequence such that $k<a_{k}$ for each $k \in \omega$. Then there exists $I \subseteq \omega$ such that:

(i) $\left\{a_{k}: k \in I\right\} \in p$;

(ii) $\left\{\left[k, a_{k}\right]: k \in I\right\}$ are pairwise disjoint intervals of $\omega$.

LEMMA $3.5([27])$. Let $p_{0}$ and $p_{1}$ be incomparable selective ultrafilters. Let $\left\{a_{k}^{j}: k \in \omega\right\} \in p_{j}$ be an increasing sequence such that $k<a_{k}^{j}$ for each $k \in \omega$ and $j \in 2$. Then there exist subsets $I_{0}$ and $I_{1}$ of $\omega$ such that:

(i) $\left\{a_{k}^{j}: k \in I_{j}\right\} \in p_{j}$ for each $j \in 2$;

(ii) $\left\{\left[k, a_{k}^{j}\right]: j \in 2\right.$ and $\left.k \in I_{j}\right\}$ are pairwise disjoint intervals of $\omega$.

LEMma 3.6. Let $\left\{p_{j}: j \in \omega\right\}$ be incomparable selective ultrafilters. Let $\left\{a_{k}^{j}: k \in \omega\right\} \in p_{j}$ be an increasing sequence such that $k<a_{k}^{j}$ for each $k, j \in \omega$. Then there exists a family $\left\{I_{j}: j \in \omega\right\}$ of subsets of $\omega$ such that:

(i) $\left\{a_{k}^{j}: k \in I_{j}\right\} \in p_{j}$ for each $j \in \omega$;

(ii) $\left\{\left[k, a_{k}^{j}\right]: j \in \omega\right.$ and $\left.k \in I_{j}\right\}$ are pairwise disjoint intervals of $\omega$.

Proof. By induction, we can construct a subset $I_{j, t}$ of $\omega$ for each $j, t \in \omega$ such that:

(a) $I_{j, t+1} \subseteq I_{j, t}$ for each $t \in \omega$;

(b) $\left\{\left[k, a_{k}^{j}\right]: j \leq t\right.$ and $\left.k \in I_{j, t}\right\}$ are pairwise disjoint intervals of $\omega$ for each $t$;

(c) $\left\{a_{k}^{j}: k \in I_{j, t}\right\} \in p_{j}$ for each $j, t \in \omega$.

From Lemma 3.5, there exists $I_{0,0} \subseteq \omega$ such that $\left\{a_{k}^{0}: k \in I_{0,0}\right\} \in p_{0}$ and $\left\{\left[k, a_{k}^{j}\right]: k \in I_{0,0}\right\}$ are pairwise disjoint.

Then conditions (a)-(c) are clearly satisfied for $j, t \leq 0$. Suppose that $I_{j, t}$ has been defined for each $j, t \leq s$ satisfying conditions (a)-(c). Let $I_{s+1,0}=\omega$ and applying Lemma $3.5 s$ times, define by induction $I_{s+1, t+1}$ and $I_{t, s+1}$ for each $t \leq s$ in such a way that: 
(1) $I_{t, s+1} \subseteq I_{t, s}$ and $I_{s+1, t+1} \subseteq I_{s+1, t}$ for each $t \leq s$;

(2) $\left\{\left[k, a_{k}^{t}\right]: k \in I_{t, s+1}\right\} \cup\left\{\left[k, a_{k}^{s+1}\right]: k \in I_{s+1, t+1}\right\}$ are pairwise disjoint intervals of $\omega$;

(3) $\left\{a_{k}^{t}: k \in I_{t, s+1}\right\} \in p_{t}$ and $\left\{a_{k}^{s+1}: k \in I_{s+1, t+1}\right\} \in p_{s+1}$ for each $t \leq s$. Clearly (a)-(c) are satisfied for each $j, t \leq s+1$. Thus, $I_{j, t}$ can be defined for each $j, t \in \omega$.

For each $j \in \omega, p_{j}$ is a $P$-point. By (c), there exists $K_{j}$ such that:

(d) $K_{j} \subseteq I_{j, j}$ and $K_{j} \backslash I_{j, t}$ is finite for each $t \in \omega$;

(e) $\left\{a_{k}^{j}: k \in K_{j}\right\} \in p_{j}$.

By (d), there exists $M_{j} \in \omega$ such that

(f) $K_{l} \subseteq I_{l, j} \cup M_{j}$ for each $l \leq j$.

Define

(g) $I_{j}=K_{j} \backslash \max \left\{a_{k}^{l}+1: l<j \wedge k<M_{j}\right\}$.

The set $K_{j} \backslash I_{j}$ is finite. By (e), the set $I_{j}$ satisfies (i) for each $j \in \omega$.

We claim that the $I_{j}$ 's also satisfy (ii). Let $j, j^{\prime}, k, k^{\prime} \in \omega$ with $k \in I_{j}$ and $k^{\prime} \in I_{j^{\prime}}$. If $j=j^{\prime}$ then by (d) and (g) it follows that $k, k^{\prime} \in I_{j} \subseteq I_{j, j}$. By (a) and (b), it follows that $\left[k, a_{k}^{j}\right] \cap\left[k^{\prime}, a_{k^{\prime}}^{j}\right]=\emptyset$. Without loss of generality, assume that $j^{\prime}<j$. It follows by construction that $k \in I_{j, j}$. If $k^{\prime} \in I_{j^{\prime}, j}$ then, by (b), we have $\left[k, a_{k}^{j}\right] \cap\left[k^{\prime}, a_{k^{\prime}}^{j^{\prime}}\right]=\emptyset$. If $k^{\prime} \notin I_{j^{\prime}, j}$ then, by (f), $k^{\prime} \in M_{j}$. By (g), it follows that $k>a_{k^{\prime}}^{j^{\prime}}$. Therefore, $\left[k, a_{k}^{j}\right] \cap\left[k^{\prime}, a_{k^{\prime}}^{j^{\prime}}\right]=\emptyset$ and condition (ii) holds.

The proof of Lemma 3.7 below does not differ substantially from a proof in [27]. In [27], Lemma 3.5 was used instead of Lemma 3.6. We give the proof for the sake of completeness.

Lemma 3.7. Let $\left\{p_{j}: j<\omega\right\}$ be incomparable selective ultrafilters, $F$ be a finite subset of $\kappa, E$ be a countable subset of $\kappa$ containing $F$, and $\left\{g_{\xi}: \xi \in E\right\}$ be a family of functions from $\omega$ into $[E]^{<\omega}$. If $\left\{S_{j}: j \in \omega\right\}$ is a family of subsets of $E$ such that $\left\{\left[g_{\xi}\right]_{p_{j}}: \xi \in S_{j}\right\} \cup\left\{[\vec{\mu}]_{p_{j}}: \mu \in E\right\}$ is linearly independent in $\left([E]^{<\omega}\right)^{\omega} / p_{j}$ for each $j \in \omega$, then there exists an increasing sequence $\left\{b_{i}: i \in \omega\right\} \subseteq \omega$, a function $r$ from $\omega$ into $\omega$ and $a$ sequence $\left\{E_{i}: i \in \omega\right\}$ of finite subsets of $E$ such that:

(a) $F \subseteq E_{0}$;

(b) $E=\bigcup_{i \in \omega} E_{i}$;

(c) $E_{i+1} \supseteq \bigcup\left\{g_{\xi}\left(b_{i}\right): \xi \in E_{i}\right\} \cup E_{i}$ for each $i \in \omega$;

(d) $\left\{g_{\xi}\left(b_{i}\right): \xi \in E_{i} \cap S_{r(i)}\right\} \cup\left\{\{\mu\}: \mu \in E_{i}\right\}$ is linearly independent for each $i \in \omega$;

(e) $\left\{b_{k}: k \in r^{-1}(j)\right\} \in p_{j}$ for each $j \in \omega$. 
Furthermore, if $\left\{y_{n}: n \in \omega\right\} \subseteq E$ is faithfully indexed, then $E_{i}$ can be arranged for each $i \in \omega$ so that

(f) $\left\{n \in \omega: y_{n} \in E_{i}\right\}=2 N$ for some $N \in \omega$.

Proof. We will first define a family $\left\{F_{n}: n \in \omega\right\}$ of finite subsets of $E$. This family will be used to construct the family $\left\{E_{n}: n \in \omega\right\}$. Define

(0) $F_{0}:=F$

and by induction on $\omega$, choose a finite subset $F_{n+1}$ of $E$ such that:

(1) $F_{n+1} \supseteq \bigcup\left\{g_{\beta}(m): m \leq n, \beta \in F_{n}\right\} \cup F_{n}$;

(2) $E=\bigcup_{n \in \omega} F_{n}$.

Let $A_{n}^{j}$ be the set $\left\{k \in \omega:\left\{g_{\xi}(k): \xi \in F_{n} \cap S_{j}\right\} \cup\left\{\{\mu\}: \mu \in F_{n}\right\}\right.$ is l.i. $\}$ for each $j, n \in \omega$. By hypothesis, $\left\{\left[g_{\xi}\right]_{p_{j}}: \xi \in S_{j}\right\} \cup\left\{[\vec{\mu}]_{p_{j}}: \mu \in E\right\}$ is linearly independent. Thus, $A_{n}^{j} \in p_{j}$ for each $n \in \omega$ and $j \in \omega$.

Selective ultrafilters are $P$-points, thus there exists $A_{j}$ such that

(3) $A_{j} \in p_{j}$ and $A_{j} \backslash A_{n}^{j}$ is finite for each $j \in \omega$ and $n \in \omega$.

Let $h_{j}: \omega \rightarrow \omega$ be an increasing function such that

(4) $A_{j} \backslash A_{n}^{j} \subseteq h_{j}(n)$ for each $j \in \omega$.

By the selectivity of $p_{j}$, there exists $B_{j}$ for each $j \in \omega$ such that

(5) $B_{j} \cap h_{j}(1)=\emptyset, B_{j} \subseteq A_{j}, B_{j} \in p_{j}$ and $\left|\left[h_{j}(n)+1, h_{j}(n+1)\right] \cap B_{j}\right| \leq 1$ for each $j \in \omega$ and $n \in \omega$.

Let $\left\{a_{n}^{j}: n \in \omega\right\}$ be the increasing enumeration of $B_{j}$ for each $j \in \omega$. From (5), it follows that $a_{n}^{j}>h_{j}(n)$ for each $j<\omega$ and $n \in \omega$. Thus, it follows from (4) that

(6) $n<a_{n}^{j}$ and $a_{n}^{j} \in A_{n}^{j}$ for each $j<\omega$ and $n \in \omega$.

Therefore, the sequences $\left\{a_{n}^{j}: n \in \omega\right\}$ for $j \in \omega$ satisfy the conditions of Lemma 3.6. By that lemma, there exists a family $\left\{I_{j}: j \in \omega\right\}$ of subsets of $\omega$ satisfying:

(7) $\left\{a_{k}^{j}: k \in I_{j}\right\} \in p_{j}$ for each $j \in \omega$;

(8) $\left\{\left[k, a_{k}^{j}\right]: j \in \omega\right.$ and $\left.k \in I_{j}\right\}$ are pairwise disjoint intervals of $\omega$.

Property (2) and $\omega \subseteq E$ imply that $\left\{I_{j}: j \in \omega\right\}$ are pairwise disjoint. Let $\left\{i_{m}: m \in \omega\right\}$ be the increasing enumeration of $\bigcup_{j<\omega} I_{j}$ and let $r: \omega \rightarrow \omega$ be such that $r(m)=j$ if and only if $i_{m} \in I_{j}$. The function $r$ is well defined as the $I_{j}$ 's are pairwise disjoint.

Define $b_{m}:=a_{i_{m}}^{r(m)}$ and $E_{m}:=F_{i_{m}}$, for each $m \in \omega$.

Properties (0) and (2) imply respectively that conditions (a) and (b) are satisfied. Condition (e) follows from the definition of $r$ and (7). 
Condition (d) is satisfied. In fact, $b_{m}=a_{i_{m}}^{r(m)} \in A_{i_{m}}^{r(m)}$ for each $m \in \omega$ by (6). By the definition of $A_{i_{m}}^{r(m)}$, the family $\left\{g_{\xi}\left(b_{m}\right): \xi \in E_{m} \cap S_{r(m)}\right\} \cup$ $\left\{\{\mu\}: \mu \in E_{m}\right\}=\left\{g_{\xi}\left(b_{m}\right): \xi \in F_{i_{m}} \cap S_{r(m)}\right\} \cup\left\{\{\mu\}: \mu \in F_{i_{m}}\right\}$ is linearly independent for each $m \in \omega$.

Finally, we check condition (c). By (8), $b_{m}=a_{i_{m}}^{r(m)} \leq i_{m+1}-1$ and $E_{m}=F_{i_{m}} \subseteq F_{i_{m+1}-1}$ for each $m \in \omega$. Thus,

$E_{m} \cup\left\{g_{\xi}\left(b_{m}\right): \xi \in E_{m}\right\}$

$$
\subseteq F_{i_{m+1}-1} \cup \bigcup\left\{g_{\xi}(k): k \leq i_{m+1}-1, \xi \in F_{i_{m+1}-1}\right\} \subseteq F_{i_{m+1}}=E_{m+1}
$$

for each $m \in \omega$. Thus, condition (c) is also satisfied and the proof is complete for items (a)-(e).

If $\left\{y_{n}: n \in \omega\right\} \subseteq E$ is faithfully indexed, it suffices to construct $\left\{F_{i}\right.$ : $i \in \omega\}$ which in addition satisfies, for each $i \in \omega,\left\{n \in \omega: y_{n} \in F_{i}\right\}=2 N$ for some $N \in \omega$. The $E_{i}$ 's clearly satisfy condition (f).

4. Finite powers of a countably compact group. Tomita [25] answered Comfort's question in the affirmative for each finite cardinal using $\mathrm{MA}_{\text {countable. }}$. We will improve these results in this section using the existence of $\mathfrak{c}$ selective ultrafilters. This is achieved by the use of Lemma 4.1. This lemma shows that the existence of selective ultrafilters implies the existence of the homomorphisms necessary to obtain the examples.

We will start with the case $n=2$. The example will be obtained by embedding $[\mathfrak{c}]^{<\omega}$ into $2^{\mathfrak{c}}$. We will first construct in Lemma 4.1 a family of homomorphisms that separate points (condition (i)), preserve promises that certain sequences will have pre-assigned $p$-limits (condition (ii)) and witness that a pre-assigned sequence of pairs has no accumulation points (condition (iii)).

Lemma 4.1. Let $\left\{p_{\xi}: \xi<\mathfrak{c}\right\}$ be incomparable selective ultrafilters. Let $F_{0}$ and $F_{1}$ be two finite subsets of $\mathfrak{c}$ and $\left\{g_{\xi}: \xi<\mathfrak{c}\right\} \subseteq\left([\mathfrak{c}]^{<\omega}\right)^{\omega}$ be such that $\bigcup_{n \in \omega} g_{\xi}(n) \subseteq \max \{\omega, \xi\}$ and $g_{\xi}$ is a one-to-one function for each $\xi<\mathfrak{c}$. Then there is a homomorphism $\Phi:[\mathfrak{c}]^{<\omega} \rightarrow 2$ such that:

(i) $\Phi\left(F^{j}\right)=1$ if $j<2$ and $F^{j} \neq \emptyset$;

(ii) $\left\{n \in \omega: \Phi\left(g_{\xi}(n)\right)=\Phi(\{\xi\})\right\} \in p_{\xi}$ for each $\xi \in \mathfrak{c}$;

(iii) $\left\{n \in \omega:(\Phi(\{2 n\}), \Phi(\{2 n+1\}))=\left(\Phi\left(F^{0}\right), \Phi\left(F^{1}\right)\right)\right\}$ is finite.

Proof. Let $F$ be the set $F^{0} \cup F^{1}$. There exists a countable subset $E$ of $\mathfrak{c}$ such that $F \cup \omega \subseteq E$ and $g_{\xi}(n) \subseteq E$ for each $\xi \in E$ and $n \in \omega$. Apply Lemma 3.7 to $\left\{p_{\xi}: \xi \in E\right\}, F, E,\left\{g_{\xi}: \xi \in E\right\}$ and $\{\{\xi\}: \xi \in E\}$ to obtain $\left\{b_{i}: i \in \omega\right\}, r: \omega \rightarrow E$ and $\left\{E_{i}: i \in \omega\right\}$ that satisfy:

(0) for each $i \in \omega, E_{i} \cap \omega=2 N$ for some $N \in \omega$;

(1) $F \subseteq E_{0}$; 
(2) $E=\bigcup_{i \in \omega} E_{i}$;

(3) $E_{i+1} \supseteq \bigcup\left\{g_{\xi}\left(b_{i}\right): \xi \in E_{i}\right\} \cup E_{i}$ for each $i \in \omega$;

(4) $\left\{g_{r(i)}\left(b_{i}\right)\right\} \cup\left\{\{\mu\}: \mu \in E_{i}\right\}$ is linearly independent for each $i \in \omega$;

(5) $\left\{b_{i}: i \in r^{-1}(\xi)\right\} \in p_{\xi}$ for each $\xi \in E$.

We will first define $\Phi$ on $[E]^{<\omega}$. This is done by an induction of length $\omega$.

For each $j<2$, let $\Phi\left(F^{j}\right)=1$ if $F^{j} \neq \emptyset$ and extend $\Phi$ to a homomorphism in $\left[E_{0}\right]^{<\omega}$. Property (i) will be satisfied no matter how we extend the homomorphism $\Phi$.

We want to construct $\Phi$ on the subgroup $\left[E_{n}\right]^{<\omega}$ for each $n \in \omega$ satisfying:

(6) $\Phi\left(g_{r(m)}\left(b_{m}\right)\right)=\Phi(\{r(m)\})$ for all $m<n$;

(7) $(\Phi(\{2 k\}), \Phi(\{2 k+1\})) \neq\left(\Phi\left(F_{0}\right), \Phi\left(F_{1}\right)\right)$ for each $m<n$ and $2 k \in$ $\left(E_{m+1} \backslash E_{m}\right) \cap \omega$.

Conditions (6) and (7) are trivially satisfied for $n=0$. Suppose that they are satisfied by $n$. We will show that $\Phi$ can be extended to $\left[E_{n+1}\right]^{<\omega}$ so that they hold for $n+1$. By (4), the set $\left\{g_{r(n)}\left(b_{n}\right)\right\} \cup\left\{\{\mu\}: \mu \in E_{n}\right\}$ is linearly independent. For each $2 k \in E_{n+1} \backslash E_{n}$, we can choose $t_{k} \in 2$ such that $\left\{g_{r(n)}\left(b_{n}\right)\right\} \cup\left\{\left\{2 k+t_{k}\right\}: 2 k \in E_{n+1} \backslash E_{n}\right\} \cup\left\{\{\mu\}: \mu \in E_{n}\right\}$ is linearly independent. Indeed, $\left\{\{\mu\}: \mu \in E_{n}\right\} \cup\left\{\{2 k+j\}: 2 k+j \in \omega \cap\left(E_{n+1} \backslash E_{n}\right)\right\}$ and $\left\{\{\mu\}: \mu \in E_{n}\right\} \cup\left\{g_{r(n)}\left(b_{n}\right)\right\}$ are linearly independent. Therefore, there exists $m \in \omega \cap\left(E_{n+1} \backslash E_{n}\right)$ such that $\left\{\{\mu\}: \mu \in E_{n}\right\} \cup\{\{2 k+j\}: 2 k+j \in$ $\left.(\omega \backslash\{m\}) \cap\left(E_{n+1} \backslash E_{n}\right)\right\} \cup\left\{g_{r(n)}\left(b_{n}\right)\right\}$ is linearly independent.

By $(3)$, we can extend $\left.\Phi\right|_{\left[E_{n}\right]<\omega}$ to $\left[E_{n+1}\right]^{<\omega}$ so that $\Phi\left(g_{r(n)}\left(b_{n}\right)\right)=$ $\Phi(\{r(n)\})$ and $\Phi\left(\left\{2 k+t_{k}\right\}\right)=1-\Phi\left(F_{t_{k}}\right)$. Therefore, (6) and (7) are satisfied by $n+1$.

Property (2) and the definition of $E$ imply that if $k \in \omega \backslash E_{0}$ then there exists $n \in \omega$ such that $2 k \in E_{n+1} \backslash E_{n}$. Property (7) implies that $\{k \in \omega$ : $\left.(\Phi(\{2 k\}), \Phi(\{2 k+1\}))=\left(\Phi\left(F^{0}\right), \Phi\left(F^{1}\right)\right)\right\} \subseteq E_{0}$. Thus, any extension of the homomorphism $\Phi$ to $[\mathfrak{c}]^{<\omega}$ satisfies condition (iii).

We claim that $\Phi$ satisfies condition (ii) for each $\xi \in E$. Indeed, by (2), given $\xi \in E$, there exists $n \in \omega$ such that $\xi \in E_{n+1} \backslash E_{n}$. By $(6), \Phi(\{\xi\})=$ $\Phi\left(g_{\xi}\left(b_{m}\right)\right)$ for each $m \in r^{-1}(\{\xi\}) \backslash n$. It follows from $r^{-1}(\{\xi\}) \backslash n \in p_{j}$ that $\xi$ satisfies condition (ii). Conditions (i) and (iii) are satisfied by any extension of $\Phi$. The construction will be finished if we extend $\left.\Phi\right|_{[E]<\omega}$ to $[\mathfrak{c}]^{<\omega}$ so that (ii) is satisfied for each $\xi \in \mathfrak{c} \backslash E$. By induction on $\mathfrak{c} \backslash E$, we will define $\Phi$ on $[E \cup \gamma]^{<\omega}$ so that (ii) is satisfied for each $\gamma \in \mathfrak{c} \backslash E$.

Let $\gamma<\mathfrak{c}$ be the least ordinal for which $\Phi(\{\gamma\})$ has not been defined yet. By the definition of $g_{\gamma},\left.\Phi\right|_{[E \cup \gamma]<\omega}$ is already defined. Thus, the sequence $\left\{\Phi\left(g_{\gamma}(k)\right): k \in \omega\right\}$ is already determined. The set $\{\{\gamma\}\} \cup\{\{\xi\}: \xi<\gamma\}$ is linearly independent. Thus, we can extend $\Phi$ to $[(\gamma+1) \cup E]^{<\omega}$ so that $\Phi(\{\gamma\})=p_{\gamma}-\lim \left\{\Phi\left(g_{\gamma}(k)\right): k \in \omega\right\}$. The homomorphism $\Phi$ satisfies (ii) for each $\xi \in(\gamma+1)$. 
We are ready to construct a countably compact group whose square is not countably compact:

EXAMPLE 4.2. Suppose that there exists a family $\left\{p_{\xi}: \xi<\mathfrak{c}\right\}$ of incomparable selective ultrafilters. Then there exists a countably compact topological group $G$ such that $G \times G$ is not countably compact.

Proof. Let $\left\{g_{\xi}: \xi<\mathfrak{c}\right\}$ be a subset of $\left([\mathfrak{c}]^{<\omega}\right)^{\omega}$ such that:

(1) $\bigcup_{n \in \omega} g_{\xi}(n) \subseteq \max \{\omega, \xi\}$;

(2) $g_{\xi}$ is one-to-one for each $\xi<\mathfrak{c}$ and for each $g \in\left([\mathfrak{c}]^{<\omega}\right)^{\omega}$ one-to-one, there exists $\mu<\mathfrak{c}$ such that $g=g_{\mu}$.

Let $\mathcal{C}=[\mathfrak{c}]^{<\omega} \times[\mathfrak{c}]^{<\omega}$. The set $\mathcal{C}$ will index the family of homomorphisms that we need. Apply Lemma 4.1 to the sequence $\left\{g_{\xi}: \xi<\mathfrak{c}\right\}$ and each pair $\left(F_{0}, F_{1}\right) \in \mathcal{C}$ to obtain a homomorphim $\Phi_{\left(F_{0}, F_{1}\right)}:[\mathfrak{c}]^{<\omega} \rightarrow 2$ satisfying:

(3) $\Phi_{\left(F_{0}, F_{1}\right)}\left(F_{j}\right)=1$ if $j<2$ and $F_{j} \neq \emptyset$;

(4) $\left\{n \in \omega: \Phi_{\left(F_{0}, F_{1}\right)}\left(g_{\xi}(n)\right)=\Phi_{\left(F_{0}, F_{1}\right)}(\{\xi\})\right\} \in p_{\xi}$ for each $\xi \in \mathfrak{c}$;

(5) $\left\{n \in \omega: \Phi_{\left(F_{0}, F_{1}\right)}(\{2 n+j\})=\Phi_{\left(F_{0}, F_{1}\right)}\left(F_{j}\right) \forall j<2\right\}$ is finite.

Let $\mathcal{H}:[\mathfrak{c}]^{<\omega} \rightarrow 2^{\mathcal{C}}$ be the diagonal map $\Delta_{\left(F_{0}, F_{1}\right) \in \mathcal{C}} \Phi_{\left(F_{0}, F_{1}\right)}$ defined by $\pi_{\left(F_{0}, F_{1}\right)} \circ \mathcal{H}=\Phi_{\left(F_{0}, F_{1}\right)}$ for each pair $\left(F_{0}, F_{1}\right) \in \mathcal{C}$, where $\pi_{\left(F_{0}, F_{1}\right)}: 2^{\mathcal{C}} \rightarrow 2$ is the projection map to the coordinate $\left(F_{0}, F_{1}\right)$.

Define $G:=\mathcal{H}\left([\mathfrak{c}]^{<\omega}\right)$ with the subspace topology. We claim that

(6) the homomorphism $\mathcal{H}$ is an embedding.

Indeed, for each non-empty finite subset $F$ of $\mathfrak{c}$, the pair $(F, F)$ is in $\mathcal{C}$. By $(3), \Phi_{(F, F)}(F) \neq 0$. Therefore, $\mathcal{H}(F) \neq 0 \in 2^{\mathcal{C}}$. Moreover

(7) $G$ is countably compact.

It suffices to show that each one-to-one sequence in $G$ has an accumulation point. If $\xi \in \mathfrak{c}$ and $\left(F_{0}, F_{1}\right) \in \mathcal{C}$ then $\Phi_{\left(F_{0}, F_{1}\right)}(\{\xi\})=p_{\xi^{-}} \lim \left\{\Phi_{\left(F_{0}, F_{1}\right)}\left(g_{\xi}(n)\right)\right.$ : $n \in \omega\}$ by (4). Therefore, $\left\{\mathcal{H}\left(g_{\xi}(n)\right): n \in \omega\right\}$ has $\mathcal{H}(\{\xi\})$ as $p_{\xi}$-limit for each $\xi<\mathfrak{c}$. The group $G$ is countably compact by $(2)$.

Finally,

(8) $G \times G$ is not countably compact.

We claim that the sequence $\{(\mathcal{H}(\{2 n\}), \mathcal{H}(\{2 n+1\})): n \in \omega\}$ does not have an accumulation point in $G \times G$. Let $\left(a_{0}, a_{1}\right)$ be an arbitrary element of $G \times G$. There exists $\left(F_{0}, F_{1}\right) \in \mathcal{C}$ such that $a_{j}=\mathcal{H}\left(F_{j}\right)$ for $j<2$. It follows from property (5) that

$$
\begin{aligned}
& \left\{n \in \omega: \pi_{\left(F_{0}, F_{1}\right)} \circ \mathcal{H}(\{2 n+j\})=\pi_{\left(F_{0}, F_{1}\right)}\left(a_{j}\right), j<2\right\} \\
& =\left\{n \in \omega:\left(\Phi_{\left(F_{0}, F_{1}\right)}(\{2 n\}), \Phi_{\left(F_{0}, F_{1}\right)}(\{2 n+1\})\right)=\left(\Phi_{\left(F_{0}, F_{1}\right)}\left(F_{0}\right), \Phi_{\left(F_{0}, F_{1}\right)}\left(F_{1}\right)\right)\right\}
\end{aligned}
$$


is finite. Therefore, $\left(a_{0}, a_{1}\right)$ is not an accumulation point of the sequence $\{(\mathcal{H}(\{2 n\}), \mathcal{H}(\{2 n+1\})): n \in \omega\}$ in $G \times G$.

The group $G$ is as required by (7) and (8).

Note: The group above could be chosen to be separable as well. For that, it suffices to consider $G \cap \overline{\langle\{\mathcal{H}(\{n\}): n \in \omega\}\rangle}$.

We can improve the example in [25] using the technique presented here:

ExAMPLE 4.3. Suppose that there exists a family $\left\{p_{\xi}: \xi<\mathfrak{c}\right\}$ of incomparable selective ultrafilters and let $n$ be a positive integer. Then there exists a topological group $G$ such that $G^{n}$ is countably compact but $G^{n+1}$ is not countably compact.

Proof. One can easily modify Lemma 3.7 to show that for a fixed integer $k$, for each $n \in \omega$ there exists $N \in \omega$ such that $E_{n} \cap \omega=(k+1) N$, and use this modification to replace $\mathrm{MA}_{\text {countable }}$ in the construction in [25]. We can construct a group $G$ generated by a linearly independent subset $\left\{x_{\xi}: \xi<\mathfrak{c}\right\}$ such that for every sequence $\left\{y_{n}^{i}: i<l\right\}$ of $l$-uples with $l \leq k$ and for each finite subset $A$ of $G$, if $\left\{n \in \omega:\left\{y_{n}^{i}: i<l\right\} \cup A\right.$ is l.i. $\}$ is cofinite in $\omega$ then $\left\{y_{n}^{i}: i<l\right\}$ has an accumulation point $G^{l}$. Then, by a result in [25], $G^{k}$ is countably compact. At the same time, as in [25], it is possible to make the sequence $\left\{\left\{x_{(k+1) n+i}: i<k\right\}: n \in \omega\right\}$ of $k+1$-uples have no accumulation point in $G^{k+1}$.

The example below improves the quasi $p$-compact group obtained by forcing in [11]:

EXAMPLE 4.4. If there exist $2^{\mathfrak{c}}$ incomparable selective ultrafilters then there exists a countably compact group $G$ such that $G$ is not quasi $M$-compact for any $M \in\left[\omega^{*}\right]^{<2^{\mathfrak{c}}}$.

We will need the following modification of Lemma 4.1.

LEMMA 4.5. Let $\left\{p_{\xi}: \xi<2^{\mathfrak{c}}\right\}$ be incomparable selective ultrafilters. Let $F_{0}$ and $F_{1}$ be two finite subsets of $2^{\mathfrak{c}}$ and $\left\{g_{\xi}: \xi<2^{\mathfrak{c}}\right\} \subseteq\left(\left[2^{\mathfrak{c}}\right]^{<\omega}\right)^{\omega}$ be such that $\bigcup_{n \in \omega} g_{\xi}(n) \subseteq \max \{\omega, \xi\}$ and $g_{\xi}$ is a one-to-one function for each $\xi<2^{\mathfrak{c}}$. Let $\left\{\alpha_{n}, \beta_{n}: n \in \omega\right\}$ be a faithfully indexed subset of $2^{\mathfrak{c}}$. Then there is a homomorphism $\Phi:\left[2^{\mathfrak{c}}\right]^{<\omega} \rightarrow 2$ such that:

(i) $\Phi\left(F^{j}\right)=1$ if $j<2$ and $F^{j} \neq \emptyset$;

(ii) $\left\{n \in \omega: \Phi\left(g_{\xi}(n)\right)=\Phi(\{\xi\})\right\} \in p_{\xi}$ for each $\xi \in 2^{\mathfrak{c}}$;

(iii) $\left\{n \in \omega:\left(\Phi\left(\left\{\alpha_{n}\right\}\right), \Phi\left(\left\{\beta_{n}\right\}\right)\right)=\left(\Phi\left(F^{0}\right), \Phi\left(F^{1}\right)\right)\right\}$ is finite.

Proof. It is a simple modification of the proof of Lemma 4.1.

Proof of Example 4.4. Applying Lemma 4.5, it is not difficult to modify Example 4.2 to obtain a countably compact group $G$ of size $2^{\mathfrak{c}}$ containing sequences $\left\{y_{n}^{\xi}: n \in \omega\right\}$ for each $\xi<2^{\mathfrak{c}}$ such that 
(*) $\quad\left\{\left(y_{n}^{\xi}, y_{n}^{\mu}\right): n \in \omega\right\}$ is closed and discrete for each pair $\xi<\mu<2^{\mathfrak{c}}$. Suppose that $G$ is quasi $M$-compact for some $M \subseteq \omega^{*}$. For each $\xi<2^{\mathfrak{c}}$, there exists $p_{\xi} \in M$ such that the $p_{\xi}$-limit of the sequence $\left\{y_{n}^{\xi}: n \in \omega\right\}$ is in $G$. From $(*)$, the ultrafilters $p_{\xi}$ are pairwise distinct. Therefore, $M$ must have cardinality $2^{\mathfrak{c}}$.

5. Infinite powers of a countably compact group. One of the difficulties in solving Question 477 for infinite cardinals that did not appear in the solution for finite cardinals, is to make an infinite power not countably compact while we add sufficiently many accumulation points for sequences of smaller powers.

In the case of infinite products in [11], the problem of the full product not being countably compact was solved only for the cardinal $2^{\mathfrak{c}}$. This was done by making the square of each group not countably compact and applying Ginsburg and Saks' theorem [13].

We will fix a sequence in the power that will witness the non-countable compactness of the $\kappa$ th power. We have to add accumulation points for sequences in small powers. However, these added points could make us add an accumulation point for the witness of the non-countable compactness of the $\kappa$ th power.

To avoid this problem, we will first add many accumulation points for sequences in small powers. We will then find a subgroup that keeps enough accumulation points for sequences in small powers. At the same time, we will keep the promise of the witness of the non-countable compactness of the larger power.

The accumulation points that witness the countable compactness of small powers come from $p$-limits with $p$ selective. On the other hand, the sequence in the $\kappa$ th power that will witness the non-countable compactness must avoid having $p$-limits for every free ultrafilter. It is not a problem to make the witness avoid $p$-limits for a selective ultrafilter $p$-we have some control over such $p$-limits. However, that is not enough to ensure that the sequence does not have accumulation points in the $\kappa$ th power. Because of this, we will need that the witness is "close to being a discrete subset of $\beta 2^{\mathfrak{c}}$ ".

We will consider two cases. First we will deal with the $2^{\mathfrak{c}}$ th power $(\mathrm{Ex}-$ ample 5.4) and later with smaller infinite powers (Example 5.6).

In Lemma 4.1, each ultrafilter $p_{\xi}$ was used to make a sequence have a $p_{\xi}$-limit. In Lemma 5.1 below, each ultrafilter $p_{\xi}$ will be used to make a small subgroup $p_{\xi}$-compact.

LEMMA 5.1. Let $\kappa=\kappa^{\omega}$ be an infinite cardinal and $F$ be a non-empty finite subset of $\kappa$ and $\left\{p_{\xi}: \xi<\kappa\right\}$ be incomparable selective ultrafilters. Let $\left\{g_{\xi}: \xi<\kappa\right\} \subseteq\left([\kappa]^{<\omega}\right)^{\omega}$ be such that $\bigcup_{n \in \omega} g_{\xi}(n) \subseteq \max \{\omega, \xi\}$ and 
$g_{\beta}$ is a one-to-one function for each $\beta<\kappa$. Let $\left\{A_{\xi}: \xi<\kappa\right\}$ be pairwise disjoint subsets of $\kappa$ such that $\left\{\left[g_{\beta}\right]_{p_{\xi}}: \beta \in A_{\xi}\right\} \cup\left\{[\vec{\mu}]_{p_{\xi}}: \mu<\kappa\right\}$ is linearly independent. Then there is a homomorphism $\Phi:[\kappa]^{<\omega} \rightarrow 2$ such that:

(i) $\Phi(F)=1$;

(ii) $\left\{n \in \omega: \Phi\left(g_{\beta}(n)\right)=\Phi(\{\beta\})\right\} \in p_{\xi}$ for each $\xi \in \kappa$ and $\beta \in A_{\xi}$.

Proof. There exists a countable subset $E$ of $\kappa$ such that $F \cup \omega \subseteq E$ and $g_{\xi}(n) \subseteq E$ for each $\xi \in E$ and $n \in \omega$. Define $I=\left\{\xi<\kappa: A_{\xi} \cap E \neq \emptyset\right\}$. The set $I$ is countable, since the $A_{\xi}$ 's are pairwise disjoint. Apply Lemma 3.7 to $\left\{p_{\xi}: \xi \in I\right\}, F, E,\left\{g_{\xi}: \xi \in E\right\}$ and $\left\{A_{\xi} \cap E: \xi \in I\right\}$ to obtain $\left\{b_{i}: i \in \omega\right\}$, $r: \omega \rightarrow I$ and $\left\{E_{i}: i \in \omega\right\}$ that satisfy:

(1) $F \subseteq E_{0}$;

(2) $E=\bigcup_{i \in \omega} E_{i}$

(3) $E_{i+1} \supseteq \bigcup\left\{g_{\xi}\left(b_{i}\right): \xi \in E_{i}\right\} \cup E_{i}$ for each $i \in \omega$;

(4) $\left\{g_{\beta}\left(b_{i}\right): \beta \in A_{r(i)} \cap E_{i}\right\} \cup\left\{\{\mu\}: \mu \in E_{i}\right\}$ is linearly independent for each $i \in \omega$

(5) $\left\{b_{i}: i \in r^{-1}(\xi)\right\} \in p_{\xi}$ for each $\xi \in I$.

We will first define $\Phi$ on $[E]^{<\omega}$. This is done by an induction of length $\omega$.

Let $\Phi(F)=1$ and extend $\Phi$ to a homomorphism in $\left[E_{0}\right]^{<\omega}$. In particular, $\Phi$ will already satisfy condition (i).

We will construct the homomorphism $\Phi$ on the subgroup $\left[E_{n}\right]^{<\omega}$ for each $n \in \omega$ satisfying:

(6) $\Phi\left(g_{\beta}\left(b_{m}\right)\right)=\Phi(\{\beta\})$ for all $\beta \in A_{r(m)} \cap E_{n}$ and all $m<n$.

Condition (6) is trivially satisfied for $n=0$. Suppose that it is satisfied by $n$. We will show that $\Phi$ can be extended to $\left[E_{n+1}\right]^{<\omega}$ so that (6) is satisfied by $n+1$. By (4),

(7) $\left\{g_{\beta}\left(b_{n}\right): \beta \in A_{r(n)} \cap E_{n}\right\} \cup\left\{\{\mu\}: \mu \in E_{n}\right\}$ is linearly independent.

By hypothesis, $\Phi(\{\beta\})$ is already defined for each $\beta \in A_{r(n)} \cap E_{n}$. By (3) and (7), $\Phi$ can be extended to $E_{n+1}$ so that $\Phi\left(g_{\beta}\left(b_{n}\right)\right)=\Phi(\{\beta\})$ for all $\beta \in A_{r(n)} \cap E_{n}$.

By property (2) at stage $\omega$, we have

(8) $\Phi$ is defined on $[E]<\omega$.

We claim that $\Phi$ satisfies condition (ii) for each $\xi \in I$ and $\beta \in A_{\xi} \cap E$. Indeed, fix $\xi \in I$ and $\beta \in A_{\xi} \cap E$. By (2), there exists $n \in \omega$ such that $\beta \in E_{n+1} \backslash E_{n}$. By $(6), \Phi(\{\beta\})=\Phi\left(g_{\beta}\left(b_{m}\right)\right)$ for each $m \in r^{-1}(\{\xi\}) \backslash n$. It follows from $r^{-1}(\{\xi\}) \backslash n \in p_{\xi}$ that $\beta$ satisfies condition (ii).

Condition (i) is satisfied by any extension of $\Phi$. The construction will be finished if we extend $\left.\Phi\right|_{[E]<\omega}$ to $[\kappa]^{<\omega}$ so that (ii) is satisfied for each $\xi \in \kappa$ and $\beta \in A_{\xi} \backslash E$.

We will define $\Phi$ on $[\kappa]^{<\omega}$ by induction on $\kappa \backslash E$. 
Let $\gamma<\kappa$ be the least ordinal for which $\Phi(\{\gamma\})$ has not been defined yet. By (8), $\Phi$ is defined on $[E \cup \gamma]^{<\omega}$. If $\gamma \notin A_{\mu}$ for any $\mu<\kappa$, extend $\Phi$ arbitrarily to $[E \cup(\gamma+1)]^{<\omega}$. Otherwise, let $\xi$ be the unique ordinal such that $\gamma \in A_{\xi}$. By the definition of $g_{\gamma}$, the sequence $\left\{\Phi\left(g_{\gamma}(k)\right): k \in \omega\right\}$ is already determined. The set $\{\gamma\} \cup\{\{\xi\}: \xi<\gamma\}$ is linearly independent. Thus, we can extend $\Phi$ to $[(\gamma+1) \cup E]^{<\omega}$ so that $\Phi(\{\gamma\})=p_{\xi}$-lim $\left\{\Phi\left(g_{\gamma}(k)\right): k \in \omega\right\}$. The homomorphism $\Phi$ clearly satisfies condition (ii) for each $\xi \in \kappa$ and $\beta \in A_{\xi} \cap(\gamma+1)$.

Lemma 5.2 below will be applied to make the sequences in small powers have many accumulation points.

Lemma 5.2. If there exist $2^{\mathfrak{c}}$ incomparable selective ultrafilters and $2^{<2^{\mathfrak{c}}}$ $=2^{\mathfrak{c}}$ then there exist:

(1) a sequence $\left\{p_{\xi}: \xi<2^{\mathfrak{c}}\right\}$ of pairwise incomparable selective ultrafilters;

(2) a sequence $\left\{I_{\xi}: \xi<2^{\mathfrak{c}}\right\}$ in $\left[2^{\mathfrak{c}}\right]^{<2^{\mathfrak{c}}}$ such that each $I \in\left[2^{\mathfrak{c}}\right]^{<2^{\mathfrak{c}}}$ appears $2^{\mathfrak{c}}$ times and $\sup I_{\xi}<\xi$ for each $\xi<2^{\mathfrak{c}}$

(3) a sequence $\left\{A_{\xi}: \xi<2^{\mathfrak{c}}\right\}$ in $\left[2^{\mathfrak{c}}\right]^{<2^{\mathfrak{c}}}$ such that $\max \left\{\xi, \sup A_{\mu}\right\}<$ $\min A_{\xi}$ for each $\mu<\xi<2^{\mathfrak{c}}$;

(4) an increasing sequence $\left\{\delta_{\xi}: \xi \in 2^{\mathfrak{c}}\right\}$ in $2^{\mathfrak{c}}$;

(5) a linearly independent subset $\left\{x_{\xi}: \xi<2^{\mathfrak{c}}\right\}$ of $2^{2^{\mathfrak{c}}}$ such that:

(6) the group generated by $\left\{x_{\eta}: \eta \in I_{\xi} \cup A_{\xi}\right\}$ is $p_{\xi}$-compact for each $\xi<2^{\mathrm{c}}$

(7) the sequence $\left\{x_{\delta_{\xi}+n}: n \in \omega\right\}$ is closed and discrete in the group generated by $\left\{x_{\eta}: \eta \in \delta_{\xi}+\omega\right\}$.

Proof. Enumerate $2^{\mathfrak{c}}$ selective ultrafilters as $\left\{p_{\xi}: \xi<2^{\mathfrak{c}}\right\}$ so that they are pairwise incomparable. By the fact that $2^{<2^{\mathfrak{c}}}=2^{\mathfrak{c}}$ (and consequently that $2^{\mathfrak{c}}$ is regular), there exist:

(a) a sequence $\left\{I_{\xi}: \xi<2^{\mathfrak{c}}\right\}$ in $\left[2^{\mathfrak{c}}\right]^{<2^{\mathfrak{c}}}$ such that each $I \in\left[2^{\mathfrak{c}}\right]^{<2^{\mathfrak{c}}}$ appears $2^{\mathfrak{c}}$ times;

(b) an increasing sequence $\left\{\delta_{\xi}: \xi<2^{\mathfrak{c}}\right\}$ in $2^{\mathfrak{c}}$;

(c) a sequence $\left\{A_{\xi}: \xi<2^{\mathfrak{c}}\right\}$ in $\left[2^{\mathfrak{c}}\right]^{<2^{\mathfrak{c}}}$ such that $A_{\xi}$ has cardinality $\mathfrak{c}+\left|I_{\xi}\right|^{\omega}$

satisfying

(d) $\max \left\{\xi, \sup A_{\mu}\right\}<\delta_{\xi}<\delta_{\xi}+\omega<\min A_{\xi}$ for each $\mu<\xi<2^{\mathfrak{c}}$.

In (c), we can choose $A_{\xi}$ such that the order type of $A_{\xi}$ is a cardinal for each $\xi \in 2^{\mathfrak{c}}$. Thus, we can define, for each $\xi \in 2^{\mathfrak{c}}$, 
(e) a family $\left\{f_{\mu}: \mu \in B_{\xi}\right\} \subseteq\left(\left[I_{\xi} \cup A_{\xi}\right]^{<\omega}\right)^{\omega}$ with $B_{\xi} \subseteq A_{\xi}$ such that $\bigcup_{n \in \omega} f_{\mu}(n) \subseteq \mu$ for each $\mu \in B_{\xi}$ and $\left\{\left[f_{\mu}\right]_{p_{\xi}}: \mu \in B_{\xi}\right\} \cup\{[\vec{\alpha}]: \alpha \in$ $\left.I_{\xi} \cup A_{\xi}\right\}$ is a basis for $\left(\left[I_{\xi} \cup A_{\xi}\right]^{<\omega}\right)^{\omega} / p_{\xi}$.

Applying Lemma 5.1, we obtain for each $F \in\left[2^{\mathfrak{c}}\right]^{<\omega} \backslash\{\emptyset\}$ a homomorphism $\phi_{F}:\left[2^{\mathfrak{c}}\right]^{<\omega} \rightarrow 2$ such that:

(i) $\phi_{F}(F) \neq 0$;

(ii) $p_{\xi^{-}} \lim \left\{\phi_{F}\left(f_{\mu}(n)\right): n \in \omega\right\}=\phi_{F}(\{\mu\})$ for each $\xi \in 2^{\mathfrak{c}}$ and $\mu \in B_{\xi}$.

We can construct, for each $\xi<2^{\mathfrak{c}}$ and $k \in \omega$, a homomorphism $\phi_{\delta_{\xi}, k}$ : $\left[2^{\mathfrak{c}}\right]^{<\omega} \rightarrow 2$ such that:

(iii) $\left.\phi_{\delta_{\xi}, k}\right|_{\left[\delta_{\xi}+k\right]<\omega}=0, \phi_{\delta_{\xi}, k}\left(\left\{\delta_{\xi}+m\right\}\right)=1$ for each $m \geq k$;

(iv) $\phi_{\delta_{\xi}, k}(\{\lambda\})=p_{\mu}$ - $\lim \left\{\phi_{\delta_{\xi}, k}\left(f_{\lambda}(n)\right): n \in \omega\right\}$ for each $\lambda \in A_{\mu}$ with $\min A_{\mu} \geq \delta_{\xi}+\omega$.

It follows by (iii) that

(v) $\phi_{\delta_{\xi}, k}(\{\lambda\})=p_{\mu}-\lim \left\{\phi_{\delta_{\xi}, k}\left(f_{\lambda}(n)\right): n \in \omega\right\}$ for each $\lambda \in A_{\mu}$ with $\sup A_{\mu}<\delta_{\xi}+\omega$.

It follows by (d) that

(vi) $\phi_{\delta_{\xi}, k}(\{\lambda\})=p_{\mu}-\lim \left\{\phi_{\delta_{\xi}, k}\left(f_{\lambda}(n)\right): n \in \omega\right\}$ for each $\mu<\kappa$ and $\lambda \in A_{\mu}$.

Let $\mathcal{B}$ be the family of homomorphisms constructed to satisfy items (i) and (ii) and $\mathcal{C}$ be the family of homomorphisms constructed to satisfy items (iii) and (vi). Let $\Phi:\left[2^{\mathfrak{c}}\right]^{<\omega} \rightarrow 2^{\mathcal{B} \cup \mathcal{C}}$ be the diagonal map, that is, $\Phi(F)=$ $\{\phi(F)\}_{\phi \in \mathcal{B} \cup \mathcal{C}}$. We can assume that $\Phi$ is a function from $\left[2^{\mathfrak{c}}\right]^{<\omega}$ into $2^{2^{\mathfrak{c}}}$, since $|\mathcal{B} \cup \mathcal{C}|=2^{\mathfrak{c}}$.

Define $x_{\eta}=\Phi(\{\eta\})$ for each $\eta \in 2^{\mathfrak{c}}$. The family $\left\{x_{\eta}: \eta<2^{\mathfrak{c}}\right\}$ is linearly independent by (i). In view of Lemma 2.6 , the group generated by $\left\{x_{\eta}: \eta \in\right.$ $\left.I_{\xi} \cup A_{\xi}\right\}$ is $p_{\xi}$-compact by (ii) and (vi). Thus, condition (6) is satisfied. It follows by (iii) that for each $\xi \in 2^{\mathfrak{c}}$ and $F \in\left[\delta_{\xi}+\omega\right]^{<\omega}$, there exists $k \in \omega$ such that $\phi_{\xi, k}(F)=0$ and $\left\{\phi_{\xi, k}\left(\left\{\delta_{\xi}+n\right\}\right): n \in \omega\right\}$ converges to 1 . Therefore condition (7) is also satisfied and the proof is complete.

LEMMA 5.3. Let $\left\{q_{\mu}: \mu<2^{\mathfrak{c}}\right\}$ be an enumeration of all free ultrafilters in $\omega^{*}$. Suppose that there exist $2^{\mathfrak{c}}$ selective ultrafilters and $2^{<2^{\mathfrak{c}}}=2^{\mathfrak{c}}$. There exist increasing sequences $\left\{J_{\mu}: \mu<2^{\mathfrak{c}}\right\}$ and $\left\{S_{\mu}: \mu<2^{\mathfrak{c}}\right\}$ in $\left[2^{\mathfrak{c}}\right]^{<2^{\mathfrak{c}}}$ and $\left\{x_{\xi}: \xi \in 2^{\mathfrak{c}}\right\} \subseteq 2^{2^{\mathfrak{c}}}$ linearly independent such that for each $\mu<2^{\mathfrak{c}}$ :

(a) $J_{\mu} \cap S_{\mu}=\emptyset$;

(b) the group $H_{\mu}$ generated by $\left\{x_{\eta}: \eta \in J_{\mu}\right\}$ is $p_{\xi_{\mu}}$-compact for some selective ultrafilter $p_{\xi_{\mu}}$

(c) $\left\langle\left\{x_{\eta}: \eta \in I \cup J_{\mu}\right\}\right\rangle$ is not $q_{\mu}$-compact for any $I \subseteq 2^{\mathfrak{c}} \backslash S_{\mu}$. 
Proof. Apply Lemma 5.2 to obtain $\left\{p_{\xi}: \xi<2^{\mathfrak{c}}\right\},\left\{I_{\xi}: \xi<2^{\mathfrak{c}}\right\},\left\{A_{\xi}\right.$ : $\left.\xi<2^{\mathfrak{c}}\right\},\left\{\delta_{\xi}: \xi \in 2^{\mathfrak{c}}\right\}$ and $\left\{x_{\xi}: \xi<2^{\mathfrak{c}}\right\}$ that satisfy conditions (1)-(7) of Lemma 5.2.

Suppose that $J_{\xi}$ and $S_{\xi}$ are already defined for each $\xi<\mu$ and satisfy conditions (a)-(c). Define $L_{\mu}=\bigcup_{\xi<\mu} J_{\xi}$ and $T_{\mu}=\bigcup_{\xi<\mu} S_{\xi}$. Then $L_{\mu}$ and $T_{\mu}$ are disjoint. Let $\beta_{\mu}<2^{\mathfrak{c}}$ be such that $\sup \left(L_{\mu} \cup T_{\mu}\right)<\delta_{\beta_{\mu}}$. By $(7)$, the sequence $\left\{x_{\delta_{\beta_{\mu}}+n}: n \in \omega\right\}$ is closed discrete in the group generated by $\left\{x_{\eta}: \eta<\delta_{\beta_{\mu}}+\omega\right\}$. Therefore, the sequence $\left\{x_{\delta_{\beta_{\mu}+n}}: n \in \omega\right\}$ does not have a $q_{\xi}$-limit in $\left\langle\left\{x_{\eta}: \eta<\delta_{\beta_{\mu}}+\omega\right\}\right\rangle$. Consider two cases:

I. $\left.\left\{x_{\delta_{\beta_{\mu}}+n}: n \in \omega\right\}\right\}$ has a $q_{\xi}$-limit in $\left\langle\left\{x_{\eta}: \eta<2^{\mathfrak{c}}\right\}\right\rangle$. In this case, there exists $F \in\left[2^{\mathfrak{c}}\right]^{<\omega}$ with $F \backslash\left(\delta_{\beta_{\mu}}+\omega\right) \neq \emptyset$ such that $q_{\xi^{-}}-\lim \left\{x_{\delta_{\beta_{\mu}}+n}: n \in \omega\right\}=$ $\sum_{\lambda \in F} x_{\lambda}$. Fix $\theta_{\mu} \in F \backslash\left(\delta_{\beta_{\mu}}+\omega\right)$. Then the sequence $\left\{x_{\delta_{\mu}+n}: n \in \omega\right\}$ does not have a $q_{\xi}$-limit in the group $\left\langle\left\{x_{\eta}: \eta \in J_{\xi} \cup I\right\}\right\rangle$ for any $I \subseteq 2^{\mathfrak{c}} \backslash\left\{\theta_{\mu}\right\}$.

II. $\left\{x_{\delta_{\beta_{\mu}+n}}: n \in \omega\right\}$ does not have a $q_{\xi}$-limit in $\left\langle\left\{x_{\eta}: \eta<2^{\mathfrak{c}}\right\}\right\rangle$. In this case, choose any $\theta_{\mu} \in 2^{\mathfrak{c}} \backslash\left(\delta_{\beta_{\mu}}+\omega\right)$.

In either case, by (2) and (3), there exists $\xi_{\mu}<2^{\mathfrak{c}}$ such that $\theta_{\mu}<\min A_{\xi_{\mu}}$ and $I_{\xi_{\mu}}=L_{\mu} \cup\left[\delta_{\beta_{\mu}}, \delta_{\beta_{\mu}}+\omega\right)$. By (6), the group generated by $\left\{x_{\eta}: \eta \in\right.$ $\left.L_{\mu} \cup\left[\delta_{\beta_{\mu}}, \delta_{\beta_{\mu}}+\omega\right) \cup A_{\xi_{\mu}}\right\}$ is $p_{\xi_{\mu}}$-compact.

Define $J_{\mu}=L_{\mu} \cup\left[\delta_{\beta_{\mu}}, \delta_{\beta_{\mu}}+\omega\right) \cup A_{\xi_{\mu}}$ and $S_{\mu}=T_{\mu} \cup\left\{\theta_{\mu}\right\}$. Clearly $J_{\mu}$ and $S_{\mu}$ satisfy conditions (a)-(c).

The following example shows that it is consistent that $2^{\mathfrak{c}}$ answers Comfort's question in the affirmative:

EXAMPLE 5.4. Assume that there exist $2^{\mathfrak{c}}$ selective ultrafilters and $2^{<2^{\mathfrak{c}}}$ $=2^{\mathfrak{c}}$. There exists a topological group $H$ such that $H^{\alpha}$ is countably compact for each $\alpha<2^{\mathfrak{c}}$, but $H^{2^{\mathfrak{c}}}$ is not countably compact.

Proof. Let $\left\{q_{\xi}: \xi<2^{\mathfrak{c}}\right\}$ be an enumeration of all free ultrafilters on $\omega$, and $\left\{J_{\mu}: \mu<2^{\mathfrak{c}}\right\},\left\{S_{\xi}: \xi<2^{\mathfrak{c}}\right\}$ and $\left\{x_{\xi}: \xi<2^{\mathfrak{c}}\right\}$ be sequences satisfying conditions (a)-(c) from Lemma 5.3.

Let $J=\bigcup_{\mu<2^{\mathrm{c}}} J_{\mu}$ and $S=\bigcup_{\mu<2^{\mathrm{c}}} S_{\mu}$. Let $H=\bigcup_{\mu<2^{\mathrm{c}}} H_{\mu}$ be the group generated by $\left\{x_{\xi}: \xi \in J\right\}$.

Claim 1. $H^{\alpha}$ is countably compact for each $\alpha<2^{\mathfrak{c}}$.

Indeed, let $Y=\left\{\left\{y_{n}^{\beta}: \beta \in \alpha\right\}: n \in \omega\right\}$ be a sequence in $H^{\alpha}$. Since the $H_{\xi}$ 's are increasing with $H=\bigcup_{\xi<2^{\mathfrak{c}}} H_{\xi}$ and $2^{\mathfrak{c}}$ is regular, there exists $\mu<2^{\mathfrak{c}}$ such that $y_{n}^{\beta}$ is an element of $H_{\mu}$ for each $n \in \omega$ and $\beta<\alpha$. Therefore, the sequence $Y$ has a $p_{\xi_{\mu}}$-limit in $\left(H_{\mu}\right)^{\alpha} \subseteq H^{\alpha}$. Thus, every sequence in $H^{\alpha}$ has an accumulation point.

Claim 2. $H^{2^{c}}$ is not countably compact. 
Suppose by way of contradiction that $H^{2^{c}}$ is countably compact. By Ginsburg and Saks' theorem, there exists a free ultrafilter for which $H$ is $p$-compact. Thus, there exists $\xi \in 2^{\mathfrak{c}}$ such that $H$ is $q_{\xi^{-}}$compact. But, by (c), $H$ is not $q_{\xi}$-compact, since $J \subseteq 2^{\mathfrak{c}} \backslash S \subseteq 2^{\mathfrak{c}} \backslash S_{\xi}$ and $J=J \cup J_{\xi}$.

We will now start the construction of the examples to answer Comfort's question for each $\kappa$ satisfying $\omega \leq \kappa<2^{\mathfrak{c}}$. In this case, we can require less than $2^{<2^{\mathfrak{c}}}=2^{\mathfrak{c}}$.

Lemma 5.5. Let $\alpha$ be an infinite cardinal smaller than $2^{\mathfrak{c}}$ and suppose that $2^{\mathfrak{c}}$ is a regular cardinal, there exist $2^{\mathfrak{c}}$ selective ultrafilters and $\left(2^{\mathfrak{c}}\right)^{<\alpha^{\omega}}$ $=2^{\mathfrak{c}}$. Then there exist:

(1) a sequence $\left\{p_{\xi}: \xi<2^{\mathfrak{c}}\right\}$ of pairwise incompatible selective ultrafilters;

(2) a sequence $\left\{I_{\xi}: \xi<2^{\mathfrak{c}}\right\}$ of all elements of $\left[2^{\mathfrak{c}}\right]^{<\alpha}$ such that each $I \in\left[2^{\mathfrak{c}}\right]^{<\alpha}$ appears $2^{\mathfrak{c}}$ times with $\sup I_{\xi}<\xi$ for each $\xi<2^{\mathfrak{c}}$;

(3) a sequence $\left\{A_{\xi}: \xi<2^{\mathfrak{c}}\right\}$ in $\left[2^{\mathfrak{c}}\right]^{<\alpha^{\omega}}$ such that $\max \{\alpha, \xi\}<\min A_{\xi}$ for each $\xi<2^{\mathfrak{c}}$;

(4) a linearly independent subset $\left\{x_{\xi}: \xi<2^{\mathfrak{c}}\right\}$ of $2^{2^{\mathfrak{c}}}$ such that:

(a) the group generated by $\left\{x_{\eta}: \eta \in I_{\xi} \cup A_{\xi}\right\}$ is $p_{\xi}$-compact for each $\xi<2^{\mathfrak{c}}$

(b) for every $A \subseteq \alpha$ there exists $\beta$ such that $\left\{\eta<\alpha: x_{\eta}(\beta)=1\right\}$ $=A$.

Proof. Define $p_{\xi}, I_{\xi}, A_{\xi}$ and $\left\{f_{\mu}: \mu \in A_{\xi}\right\}$ such that $\left\{\left[f_{\mu}\right]_{p_{\xi}}: \mu \in\right.$ $\left.A_{\xi}\right\} \cup\left\{[\{\mu\}]_{p_{\xi}}: \mu \in I_{\xi} \cup A_{\xi}\right\}$ is a basis for $\left(\left[I_{\xi} \cup A_{\xi}\right]^{<\omega}\right)^{\omega} / p_{\xi}$, for each $\xi \in 2^{\mathfrak{c}}$. This can be done similarly to the proof of Lemma 5.2.

As in the proof of that lemma, for each $F \in\left[2^{\mathfrak{c}}\right]^{<\omega} \backslash\{\emptyset\}$, let $\Phi_{F}:\left[2^{\mathfrak{c}}\right]^{<\omega}$ $\rightarrow 2$ be a homomorphism such that $\Phi_{F}(F) \neq 0$ and $p_{\xi}$-lim $\left\{\Phi_{F}\left(f_{\mu}(n)\right)\right.$ : $n \in \omega\}=\Phi_{F}(\{\mu\})$ for each $\xi<2^{\mathfrak{c}}$ and $\mu \in A_{\xi}$. For each $A \subseteq \alpha$, the set $\{\{\eta\}: \eta<\alpha\}$ is linearly independent. Thus, we can define a homomorphism $\Psi_{A}:[\alpha]^{<\omega} \rightarrow 2$ such that $\left\{\eta \in \alpha: \Psi_{A}(\{\eta\})=1\right\}=A$. This homomorphism can be extended to $\left[2^{\mathfrak{c}}\right]^{<\omega}$ in such a way that $p_{\xi^{-}} \lim \left\{\Psi_{A}\left(f_{\mu}(n)\right): n \in \omega\right\}=$ $\Psi_{A}(\{\mu\})$ for each $\xi<2^{\mathfrak{c}}$ and $\mu \in A_{\xi}$.

Then $x_{\eta}=\left\{\Phi_{F}(\{\eta\}): F \in\left[2^{\mathfrak{c}}\right]^{<\omega} \backslash\{\emptyset\}\right\} \cup\left\{\Psi_{A}(\{\eta\}): A \subseteq \alpha\right\}$ for each $\eta \in 2^{\mathfrak{c}}$ are as required.

ExAmPle 5.6. Assume that $2^{\mathfrak{c}}$ is a regular cardinal, there exist $2^{\mathfrak{c}}$ incomparable selective ultrafilters and $\left(2^{\mathfrak{c}}\right)^{<\alpha^{\omega}}=2^{\mathfrak{c}}$. There exists a topological group $H$ such that $H^{\gamma}$ is countably compact for each $\gamma<\alpha$, but $H^{\alpha}$ is not countably compact.

Proof. Let $\left\{p_{\xi}: \xi<2^{\mathfrak{c}}\right\},\left\{I_{\xi}: \xi<2^{\mathfrak{c}}\right\},\left\{A_{\xi}: \xi<2^{\mathfrak{c}}\right\}$ and $\left\{x_{\xi}: \xi<2^{\mathfrak{c}}\right\}$ be such that (1)-(4) and (a)-(b) in Lemma 5.5 are satisfied. 
It follows by (b) that

(c) for any distinct $p, q \in \omega^{*}, k_{0}, k_{1}, l_{0}, l_{1} \in 2$ with $\left(k_{0}, k_{1}\right) \neq(0,0)$ or $\left(l_{0}, l_{1}\right) \neq(0,0)$ and $\theta_{0}, \theta_{1}, \lambda_{0}, \lambda_{1}$ limit ordinals below $\alpha$,

$p-\lim \left\{k_{0} x_{\theta_{0}+n}+k_{1} x_{\theta_{1}+n}: n \in \omega\right\} \neq q-\lim \left\{l_{0} x_{\lambda_{0}+n}+l_{1} x_{\lambda_{1}+n}: n \in \omega\right\}$.

Let $\left\{g_{\xi}: \xi<2^{\mathfrak{c}}\right\}$ be an enumeration of all $g$ such that $\operatorname{dom} g=F$ with $F \in\left[2^{\mathfrak{c}}\right]^{<\alpha}$ and $g(\mu): \omega \rightarrow\left[2^{\mathfrak{c}}\right]^{<\omega}$ for all $\mu \in F$. Let $\mathcal{P}$ be the set of all $p \in \omega^{*}$ such that $p$ - $\lim \left\{x_{\theta+n}: n \in \omega\right\} \in\left\langle\left\{x_{\eta}: \eta<2^{\mathfrak{c}}\right\}\right\rangle$ for each $\theta<\alpha$ limit.

We will define by induction $\left\{K_{\xi}: \xi<2^{\mathfrak{c}}\right\},\left\{P_{\xi}: \xi<2^{\mathfrak{c}}\right\},\left\{S_{\xi}: \xi<2^{\mathfrak{c}}\right\}$, $\left\{\lambda_{\xi}: \xi<2^{\mathfrak{c}}\right\}$ and $\left\{\gamma_{\xi}: \xi<2^{\mathfrak{c}}\right\}$ such that:

(i) $K_{\xi} \in\left[2^{\mathfrak{c}}\right]^{<\alpha}$;

(ii) $\gamma_{\xi}$ is the least ordinal $\gamma<2^{\mathfrak{c}}$ such that $\gamma \notin\left\{\gamma_{\eta}: \eta<\xi\right\}$ and $\bigcup_{\mu \in \operatorname{dom} g_{\gamma} \wedge n \in \omega} g_{\gamma}(\mu)(n) \subseteq \alpha \cup \bigcup_{\mu<\xi} K_{\mu}$

(iii) $\lambda_{\xi} \in 2^{\mathfrak{c}}$ for each $\xi<2^{\mathfrak{c}}$;

(iv) $p_{\lambda_{\xi}}-\lim \left\{x_{g_{\xi}(\mu)(n)}: n \in \omega\right\} \in\left\langle\left\{x_{\eta}: \eta \in \alpha \cup \bigcup_{\nu \leq \xi} K_{\nu}\right\}\right\rangle$ for each $\mu \in \operatorname{dom} g_{\xi}$

(v) $P_{\xi} \subseteq \omega^{*}$ is the set of all ultrafilters $p \in \mathcal{P}$ such that $p$ - $\lim \left\{y_{n}\right.$ : $n \in \omega\} \in\left\langle\left\{x_{\eta}: \eta \in \alpha \cup \bigcup_{\mu<\xi} K_{\mu}\right\}\right\rangle$, where $\left\{y_{n}: n \in \omega\right\}$ is a sequence of the form $\left\{x_{\beta+n}: n \in \omega\right\}$ for some $\beta<\alpha$ limit or $\left\{x_{\{\beta+n, \zeta+n\}}: n \in \omega\right\}$ with $\beta<\zeta<\alpha$ limit ordinals;

(vi) for every $p \in P_{\xi}$, there exists a limit ordinal $\beta<\alpha$ such that the $p$-limit of the sequence $\left\{x_{\beta+n}: n \in \omega\right\}$ does not belong to $\left\langle\left\{x_{\eta}: \eta \in 2^{\mathfrak{c}} \backslash S_{\xi}\right\}\right\rangle ;$

(vii) $\left|P_{\xi}\right| \leq|\xi|+\alpha$ and $\left|S_{\xi}\right| \leq|\xi|+\alpha$;

(viii) $\left(\bigcup_{\mu \leq \xi} K_{\mu}\right) \cap S_{\xi}=\alpha \cap S_{\xi}=\alpha \cap K_{\xi}=\emptyset$;

(ix) $P_{\gamma} \subseteq P_{\xi}$ for each $\gamma<\xi$ and if $\xi$ is a limit ordinal then $P_{\xi}=$ $\bigcup_{\gamma<\xi} P_{\gamma}$

(x) $S_{\gamma} \subseteq S_{\xi}$ for each $\gamma<\xi$ and if $\xi$ is a limit ordinal then $S_{\xi}=$ $\bigcup_{\gamma<\xi} S_{\gamma}$.

At stage 0 , start with $K_{0}=\emptyset$. We note first that $P_{0}=\emptyset$. Indeed, let $F \in[\alpha]^{<\omega}$ and $\beta<\alpha$ be limit ordinals. By (b), there exists $\mu<2^{\mathfrak{c}}$ such that $\left\{\eta<\alpha: x_{\eta}(\mu)=1\right\}=[\beta, \beta+\omega) \backslash F$. Then $x_{F}(\mu)=0$ is not an accumulation point of the sequence $\left\{x_{\beta+n}(\mu): n \in \omega\right\}$ or the sequence $\left\{x_{\{\beta+n, \zeta+n\}}(\mu): n \in \omega\right\}$ for any $\zeta<\alpha$ limit with $\beta \neq \zeta$.

Define $S_{0}=\emptyset$. Then conditions (i)-(x) are satisfied.

Suppose by induction that $\left\{P_{\mu}: \mu<\beta\right\},\left\{S_{\mu}: \mu<\beta\right\},\left\{K_{\mu}: \mu<\eta<\beta\right\}$, $\left\{\gamma_{\mu}: \mu<\eta<\beta\right\}$ and $\left\{\lambda_{\mu}: \mu<\eta<\beta\right\}$ are defined.

If $\beta$ is limit, let $P_{\beta}=\bigcup_{\mu<\beta} P_{\mu}$ and $S_{\beta}=\bigcup_{\mu<\beta} S_{\mu}$. Then conditions (ix) and $(\mathrm{x})$ are satisfied. 
We claim that condition (v) is satisfied. Let $p \in P_{\beta}$ and $\left\{y_{n}: n \in \omega\right\}$ be as in (v). There exists $F \subseteq \alpha \cup \bigcup_{\eta<\beta} K_{\eta}$ finite such that $x_{F}$ is the $p$-limit of $\left\{y_{n}: n \in \omega\right\}$. Then there exists $\mu<\beta$ such that $F \subseteq \alpha \cup \bigcup_{\eta<\mu} K_{\eta}$. Therefore, $p \in P_{\mu}$.

To check (vi), let $p \in P_{\beta}$. Then there exists $\mu<\beta$ such that $p \in P_{\mu+1} \backslash P_{\mu}$. By hypothesis, (vi) is satisfied for $\mu+1$, thus there exists $\theta<\alpha$ such that $p$ - $\lim \left\{x_{\theta+n}: n \in \omega\right\} \notin\left\langle\left\{x_{\eta}: \eta \in 2^{\mathfrak{c}} \backslash S_{\mu+1}\right\}\right\rangle$. Condition (vi) is satisfied by $\beta$, since $2^{\mathfrak{c}} \backslash S_{\beta} \subseteq 2^{\mathfrak{c}} \backslash S_{\mu+1}$. Conditions (i)-(iv) and (vii)-(viii) are clearly satisfied.

If $\beta=\mu+1$, let $\gamma_{\mu}$ be the least ordinal $\gamma$ such that

$$
\bigcup_{\eta \in \operatorname{dom} g_{\gamma} \wedge n \in \omega} g_{\gamma}(\eta)(n) \subseteq \alpha \cup \bigcup_{\xi<\mu} K_{\xi} .
$$

There exists $\lambda_{\mu}<2^{\mathfrak{c}}$ such that $A_{\lambda_{\mu}} \cap S_{\mu}=\emptyset$ and $\left\langle\left\{x_{\eta}: \eta \in \alpha \cup \bigcup_{\theta<\mu} K_{\theta} \cup\right.\right.$ $\left.\left.A_{\lambda_{\mu}}\right\}\right\rangle$ is $p_{\lambda_{\mu}}$-compact.

For each $\xi \in \operatorname{dom} g_{\gamma_{\mu}}$, there exists $F_{\xi} \subseteq A_{\lambda_{\mu}}$ finite such that $p_{\lambda_{\mu}}$ $\lim \left\{x_{g_{\gamma_{\mu}}(\xi)(n)}: n \in \omega\right\}-x_{F_{\xi}} \in\left\langle\left\{x_{\eta}: \eta \in \alpha \cup \bigcup_{\theta<\mu} K_{\theta}\right\}\right\rangle$. Let $K_{\mu}=$ $\bigcup_{\xi \in \operatorname{dom} g_{\gamma \mu}} F_{\xi}$. Then conditions (i)-(iv) are satisfied.

Let $P_{\beta}$ be as in condition (v). Condition (ix) is clearly satisfied. If $p \in P_{\beta}$ then there exists $\left\{y_{n}: n \in \omega\right\}$ as in (v) such that $a_{p}=p-\lim \left\{y_{n}: n \in \omega\right\} \in$ $\left\langle\left\{x_{\eta}: \eta \in \alpha \cup \bigcup_{\mu<\xi} K_{\mu}\right\}\right\rangle$. By (c), $a_{p} \neq a_{q}$ for distinct $p, q \in P_{\beta}$. Then $\left|P_{\beta}\right| \leq\left|\left\langle\left\{x_{\eta}: \eta \in \alpha \cup \bigcup_{\mu<\xi} K_{\mu}\right\}\right\rangle\right| \leq|\xi| . \alpha$. Thus, the first part of (vii) is satisfied.

We will define $S_{\beta}$. Fix $p \in P_{\beta} \backslash P_{\mu}$. For each $\theta<\alpha$ limit, there exists a finite subset $E_{\theta}$ of $\left[2^{\mathfrak{c}} \backslash\left(\alpha \cup \bigcup_{\xi<\mu} K_{\xi}\right)\right]^{<\omega}$ such that $p$-lim $\left\{x_{\theta+n}: n \in \omega\right\}$ $-x_{E_{\theta}} \in\left\langle\left\{x_{\eta}: \eta \in \alpha \cup \bigcup_{\xi<\mu} K_{\xi}\right\}\right\rangle$. We claim that if $\theta<\theta^{\prime}<\alpha$ are limit then $E_{\theta} \neq E_{\theta^{\prime}}$. Indeed, if $E_{\theta}=E_{\theta^{\prime}}$ then $p$-lim $\left\{x_{\left\{\theta+n, \theta^{\prime}+n\right\}}: n \in \omega\right\} \in$ $\left\langle\left\{x_{\eta}: \eta \in \alpha \cup \bigcup_{\xi<\mu} K_{\xi}\right\}\right\rangle$. Thus, $p \in P_{\mu}$, which is a contradiction. Since $\left|\left[K_{\mu}\right]^{<\omega}\right|<\alpha$, there exists $\theta_{p}<\alpha$ such that $E_{\theta_{p}} \nsubseteq K_{\mu}$. Fix $\zeta_{p} \in E_{\theta_{p}} \backslash K_{\mu}$ for each $p \in P_{\beta} \backslash P_{\mu}$.

Define $S_{\beta}=S_{\mu} \cup\left\{\zeta_{p}: p \in P_{\beta} \backslash P_{\mu}\right\}$.

Conditions (vi) and (x) are clearly satisfied. The second part of (vii) holds by the definition of $S_{\beta}$ and the first part of condition (vii). Property (viii) is satisfied by the definition of $S_{\beta}$ and the fact that $\zeta_{p} \in E_{\theta_{p}}, E_{\theta_{p}} \cap \bigcup_{\gamma<\mu} K_{\gamma}$ $=\emptyset$ and $\zeta_{p} \notin K_{\mu}$ for each $p \in P_{\beta}$.

Thus, the induction can be carried on up to $2^{\mathfrak{c}}$. Let $H$ be the group generated by $\left\{x_{\eta}: \eta \in \alpha \cup \bigcup_{\xi<2^{\mathrm{c}}} K_{\xi}\right\}$.

Claim 1. Powers of $H$ smaller than $\alpha$ are countably compact. 
Let $\mu<\alpha$ and $\left\{a_{n}^{\eta}: \eta<\mu \wedge n \in \omega\right\} \subseteq H$. Since the cofinality of $2^{\mathfrak{c}}$ is bigger than $\mu$, there exists $\beta<2^{\mathfrak{c}}$ such that $a_{n}^{\eta}=x_{g_{\gamma_{\beta}}(\eta)(n)}$ for each $\eta<\mu$ and $n \in \omega$. By condition (iv), $p_{\lambda_{\beta}} \lim \left\{a_{n}^{\eta}: n \in \omega\right\} \in\left\langle\left\{x_{\theta}: \theta \in\right.\right.$ $\left.\left.\alpha \cup \bigcup_{\xi<\beta} K_{\xi}\right\}\right\rangle \subseteq H$ for each $\eta<\mu$. Thus, $H^{\mu}$ is countably compact.

Claim 2. $H^{\alpha}$ is not countably compact.

We will show that $\left\{\left\{x_{\mu+n}: \mu<\alpha\right.\right.$ limit $\left.\}: n \in \omega\right\}$ does not have an accumulation point in $H^{\alpha}$. Indeed, if this sequence has an accumulation point, then there exists $p \in \omega^{*}$ such that $p-\lim \left\{x_{\mu+n}: n \in \omega\right\} \in H$ for each $\mu<\alpha$ limit. Then $p \in \mathcal{P}$ and there is $\xi<2^{\mathfrak{c}}$ such that $p-\lim \left\{x_{n}: n \in \omega\right\}$ $\in\left\langle\left\{x_{\eta}: \eta \in \alpha \cup \bigcup_{\mu<\xi} K_{\mu}\right\}\right\rangle$. Thus, $p \in P_{\xi+1}$. By property (v), there is $\theta<\alpha$ limit such that $p$-lim $\left\{x_{\theta+n}: n \in \omega\right\} \notin\left\langle\left\{x_{\eta}: \eta \in 2^{\mathfrak{c}} \backslash S_{\xi+1}\right\}\right\rangle \supseteq H$, which is a contradiction. Therefore, $H^{\alpha}$ is not countably compact.

Final remarks. A natural question is whether selective ultrafilters are necessary to obtain the example in this note. Another natural question is whether we can construct the examples using fewer selective ultrafilters. The following questions remain open:

QUESTION 5.7. Does the existence of $\mathfrak{c}$ Rudin-Keisler incomparable Ppoints imply the existence of countably compact topological groups whose product is not countably compact?

Question 5.8. Does the existence of a selective ultrafilter imply the existence of a topological group $G$ and an infinite cardinal $\kappa$ such that $G^{\lambda}$ is countably compact for all cardinals $\lambda<\kappa$, but $G^{\kappa}$ is not countably compact?

The first ideas for this paper started to take form during the author's visit to Prof. Tsugunori Noguri at Ehime University in 2002. The author acknowledges the support of Ministry of Education of Japan for this visit. The author would like to express his gratitude to Sampo and Hiroko Ueda for their support and help during the author's visit to Matsuyama.

The author would like to thank Marina Paesano for her help during the time the research that led to this paper was done. The author thanks Gordon Sakang Sone and Diane Castonguay for revising the English of the text.

The author acknowledges the support of UMALCA to present this work at the III JAMEX in Oaxaca, Mexico.

\section{References}

[1] J. Baumgartner, Sacks forcing and the total failure of Martin's Axiom, Topology Appl. 19 (1985), 211-225.

[2] A. R. Bernstein, A new kind of compactness for topological spaces, Fund. Math. 66 (1970), 185-193. 
[3] A. R. Blass and S. Shelah, There may be $P_{\aleph_{1}}$-points and $P_{\aleph_{2}}$-points and the RudinKeisler order may be downward directed, Ann. Pure Appl. Logic 33 (1987), 213-243.

[4] W. W. Comfort, Problems on topological groups and other homogeneous spaces, in: Open Problems in Topology, J. van Mill and G. M. Reed (eds.), North-Holland, 1990, 311-347.

[5] W. W. Comfort and S. Negrepontis, The Theory of Ultrafilters, Springer, Berlin, 1974.

[6] W. W. Comfort and K. A. Ross, Pseudocompactness and uniform continuity in topological groups, Pacific J. Math. 16 (1966), 483-496.

[7] D. Dikranjan and D. Shakhmatov, Forcing hereditarily separable compact-like group topologies on Abelian groups, Topology Appl., to appear.

[8] D. Dikranjan and M. Tkachenko, Algebraic structure of small countably compact Abelian groups, Forum Math. 15 (2003), 811-837.

[9] E. K. van Douwen, The product of two countably compact topological groups, Trans. Amer. Math. Soc. 262 (1980), 417-427.

[10] S. Garcia-Ferreira, Three ordering on $\beta(\omega) \backslash \omega$, Topology Appl. 50 (1993), 199-216.

[11] S. Garcia-Ferreira and A. H. Tomita, Countably compact groups and p-limits, Bol. Soc. Mex. Mat. (3) 9 (2003), 309-321.

[12] S. Garcia-Ferreira, A. H. Tomita and S. Watson, Countably compact groups from a selective ultrafilter, Proc. Amer. Math. Soc. 133 (2005), 937-943.

[13] J. Ginsburg and V. Saks, Some applications of ultrafilters in topology, Pacific J. Math. 57 (1975), 403-418.

[14] A. Hajnal and I. Juhász, A normal separable group need not be Lindelöf, Gen. Top. Appl. 6 (1976), 199-205.

[15] K. P. Hart and J. van Mill, A countably compact topological group $H$ such that $H \times H$ is not countably compact, Trans. Amer. Math. Soc. 323 (1991), 811-821.

[16] P. B. Koszmider, A. H. Tomita and S. Watson, Forcing countably compact group topologies on a larger free Abelian group, Topology Proc. 25 (2000), 563-574.

[17] J. Novák, On the cartesian product of two spaces, Fund. Math. 40 (1953), 106-112.

[18] V. Saks, Products of countably compact spaces, Topology Proc. 4, (1979), 553-575.

[19] C. T. Scarborough and A. H. Stone, Products of nearly compact spaces, Trans. Amer. Math. Soc. 124 (1966), 131-147.

[20] H. Terasaka, On Cartesian product of compact spaces, Osaka Math. J. 4 (1952), $11-15$.

[21] M. Tkachenko, On countably compact and pseudocompact topologies on free Abelian groups, Soviet Math. (Izv. VUZ) 34 (1990), no. 5, 79-86.

[22] A. H. Tomita, On finite powers of countably compact groups, Comment. Math. Univ. Carolin. 37 (1996), 617-626.

[23] - A group under $\mathrm{MA}_{\text {countable }}$ whose square is countably compact but whose cube is not, Topology Appl. 91 (1999), 91-104.

[24] - Two countably compact groups: one of size $\aleph_{\omega}$ and the other of weight $\aleph_{\omega}$ without non-trivial convergent sequences, Proc. Amer. Math. Soc. 131 (2003), 2617-2622.

[25] - Countable compactness in finite powers of topological groups without convergent sequences, Topology Appl. 146/147 (2005), 527-538.

[26] - , The weight of a countably compact group whose size has countable cofinality, ibid. 150 (2005), 197-205.

[27] A. H. Tomita and S. Watson, Ultraproducts, p-limits and antichains on the Comfort group order, ibid. 143 (2004), 147-157. 
[28] S. L. Yang, On products of countably compact groups, Topology Proc. 10 (1985), 221-230.

Departamento de Matemática

Instituto de Matemática e Estatística

Universidade de São Paulo

Caixa Postal 66281

CEP 05315-970, São Paulo, Brazil

E-mail: tomita@ime.usp.br

Received 1 July 2004;

in revised form 31 October 2004 